\title{
Identification of interturn faults in power transformers by means of generalized symmetrical components analysis
}

\author{
Daniil Ivanchenko ${ }^{1, *}$, and Artem Smirnov ${ }^{1}$ \\ ${ }^{1}$ Saint-Petersburg Mining University, Department of Electrical Engineering, 199106 St Petersburg, Russian Federation
}

\begin{abstract}
The paper deals with experimental identification of transformer internal faults, an important factor in reliability and sustainability of power supply systems. Task of identification of transformer internal faults requires increasing sensitivity of relay protection by calculation of components most sensitive to interwire faults from transformer current. In order to study internal faults in transformer, the model in Simulink MATLAB was developed on the basis of transformer constitutive equations. Transformer with short circuited wires was simulated as a multiwinding transformer. We provide the calculation of transformer parameters. Model was applied for analysis of transients in power transformers, such as interwire fault, transformer inrush, and fault in transformer connections. Analysis of power transformer internal faults by means of time-dependent symmetrical components of currents is provided. These symmetrical components were calculated for the first harmonic of current by means of discrimination of firs harmonic by low-pass filter and compensating elements implementing phase shift. Described method allows calculation of symmetrical components during transient and under nonsinusoidal conditions. Simulation results showed the advantage of instantaneous symmetrical components of other direct values. Those components were implemented in relay protection algorithms for identification of internal faults in transformers.
\end{abstract}

\section{Introduction}

Power transformers are important part of any electric power system, and significantly affect the sustainability and reliability of power supply. The transformer reliability is dependent on effective relay protection operation. Main problems in relay protection are failures in case of turn-to-turn fault in the transformer and in unjustified tripping in case of transients in energy system outside the protected zone [1], [2].

As a result of growing energy capacity of modern industry [3], [4], [5], [6], the failure of relay protection in case of interwire fault will lead significant damage caused to the transformer by fault currents and results high repair costs.

The major methods for increasing sensitivity of relay protection are calculation of components most sensitive to interwire faults from transformer currents. Examples of such components are symmetrical components, dq0 transform, wavelet transform and other [7], [8], [9], [10], [11], [12]. In order to estimate and improve algorithms of relay protection, simulation of transformer transients is required.

This study is dedicated to development of transformer model in Simulink MATLAB, which would provide calculation of transformer transients in cases of internal faults and over transients in transformers, and provide means to identify internal faults from other transients.

\section{Methods}

Internal short circuits in transformer occur if insulation of transformer windings is damaged. In case of main insulation damage ground faults take place; in case of longitudinal insulation winding end winding fault happen. Interwire fault could happen between coils of same or different phases. Short circuits between coils and wires of transformer windings are most common for early stages of transformer faults.

In cases of interwire fault transformer can be considered as multiwinding transformer [13]. Structural model of ideal single-phase transformer in case of interwire fault is presented on fig.1.

\footnotetext{
* Corresponding author: dan87332@mail.ru
} 


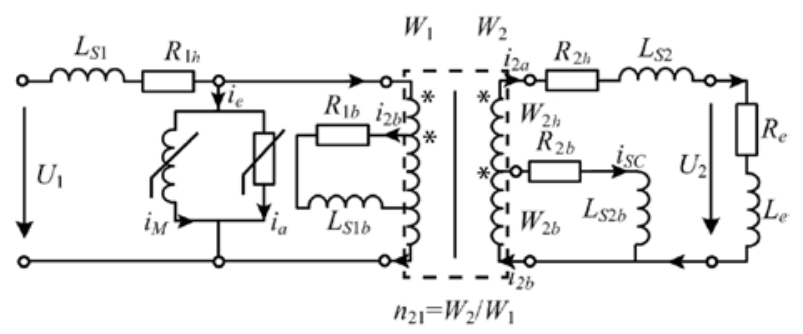

Fig. 1. Structural model of transformer with interwire fault.

\subsection{Interwire fault simulation}

In case of short circuit between wires of second winding $W_{2}$, two magnetically coupled coils are formed. First coil have number of turns $W_{2 b}=\beta W_{2}, \beta<1$ and is short circuited. Second coil have number of turns $W_{2 h}=(1-\beta) W_{2}$. Short circuit current is specified by resistance $R_{2 b}$, leakage inductance $L_{s 2 b}$, number of short-circuited turns $W_{2 b}$ and fault resistance $R_{a r c-b}$. Calculation of $L_{s 2 b}$ and $R_{2 b}$ is established by means of electromagnetic field theory [12, 13]. For a first approximation it is possible to consider, that the parameters of short circuited part if winding $W_{2 b}$ are proportional to relative number of short circuited turns:

$$
R_{2 b}=\beta R_{2} ; L_{s 2 b}=\beta L_{s 2}
$$

Where $R_{2}$ and $L_{s 2}$ - resistance and leakage inductance of secondary winding of healthy transformer, which related to parameters of short circuit $R_{s c}$ and $L_{s c}$ by formulas:

$$
R_{2}=\frac{R_{s c}}{2} \cdot n_{21}^{2} ; L_{2 s}=\frac{L_{s c}}{2} \cdot n_{21}^{2} ; n_{21}^{2}=\frac{W_{2}}{W_{1}}
$$

The current $i_{2}$ of secondary winding with number of turns $W_{2 h}=(1-\beta) \cdot W_{2}$ passes through equivalent resistance $R_{e}$ and inductance $L_{e}$ of the load. Parameters of winding $W_{2 h}$ are calculated as:

$$
L_{s 2 h}=(1-\beta) \cdot L_{s 2} ; R_{2 h}=(1-\beta) \cdot R_{2}
$$

Currents $i_{2}, i_{2 b}$ and current of short circuited mesh $i_{s c 2}$ in winding $W_{2 b}$ are calculated from Kirchhoff equations for circuit on fig.1:

$$
\begin{aligned}
& u_{\mu 2}=R_{2 h} i_{2}+L_{2 h} \cdot \frac{d i_{2}}{d t}+u_{2} ; \\
& u_{\mu 2}=n_{21} u_{\mu 1} ; u_{\mu 1}=W_{1} \cdot \frac{d \Phi_{1}}{d t} ; \\
& u_{\mu 2 b}=R_{2 b} i_{2}+L_{s 2 b} \cdot \frac{d i_{2 b}}{d t}+R_{a r c 2} i_{2} ; \\
& u_{\mu 2 b}=\beta n_{21} u_{\mu 1} ; i_{2 b}=i_{2}+i_{s c 2}
\end{aligned}
$$

Where $\Phi_{1}-$ magnetic flux of core with length $l_{c}$, on which coils $W_{1}$ and $W_{2}$ are situated, $u_{2}-$ load voltage.

\subsection{Simulink MATLAB implementation}

In accordance with previously established set of constitutive equations of transformer, structural diagram of the calculation (fig.2) was developed.

As the simulation of transients in transformer in cases of transients in electric grid is required, it those were implemented by means of SimPowerSystems BlockSet. In order to couple calculation blocks in Simulink with models, made with SimPowerSystems BlockSet library, model of transformer was connected through Controlled Current Source and Voltage Measurement blocks.

Structural diagram of connection of three-phase delta-wye transformer is represented on fig.3.Each phase of three-phase transformer represented by single-phase transformer. Subsystems Phase_A, Phase_B and Phase_C, are structural diagrams of calculation of single phase transformer constitutive equation

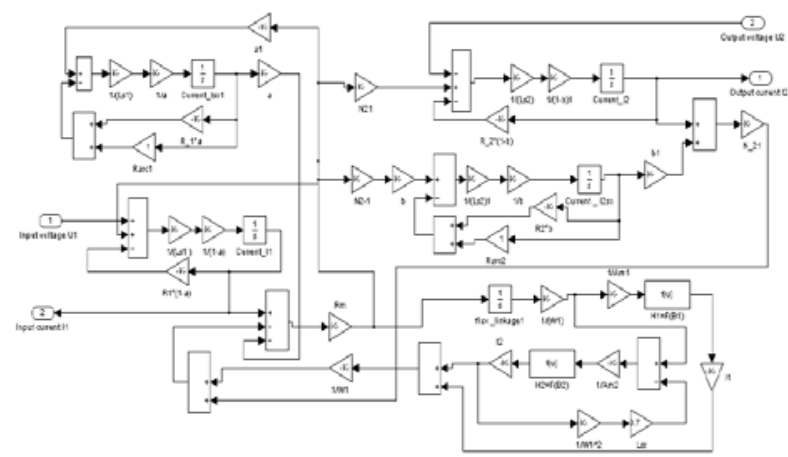

Fig. 2. Structural diagram of calculation of transformer constitutive equations. 


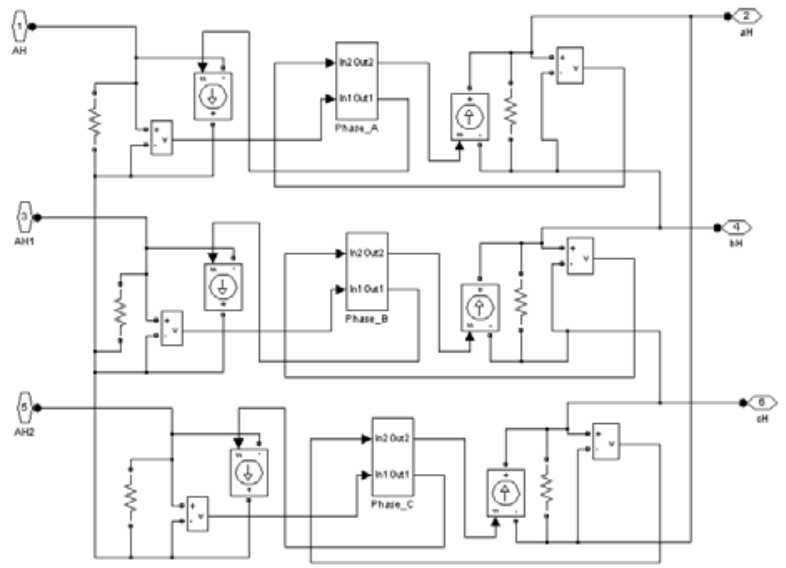

Fig. 3. Structural diagram of SimPower BlockSet connection implementation.

\subsection{Fault identification}

Generalized symmetrical components analysis [14] covers the case of nonsinusoidal and asymmetric dynamic systems of three-phase variables forming the vector: $x_{p \text { no }}=\left[x_{p}, x_{n}, x_{o}\right]^{T}$. Instantaneous components of positive and negative sequences $x_{p}(t)$ and $x_{n}(t)$ are complex variables $x_{p}=x_{p r}+j x_{p i}, x_{n}=x_{n r}+j x_{n i}$, zero sequence component is a real value $x_{o}(t)$. Symmetrical components of vector $x_{p n o}$ are calculated by this equation:

$$
\left(\begin{array}{l}
x_{p}(t) \\
x_{n}(t) \\
x_{o}(t)
\end{array}\right)=\left(\begin{array}{l}
x_{p r}(t)+j \cdot x_{p i}(t) \\
x_{p r}(t)-j \cdot x_{p i}(t) \\
x_{o}(t)
\end{array}\right)=\frac{1}{\sqrt{3}} \cdot\left[\left(\begin{array}{ccc}
1 & a & a^{2} \\
1 & a^{2} & a \\
1 & 1 & 1
\end{array}\right) \cdot\left(\begin{array}{l}
x_{a}(t) \\
x_{b}(t) \\
x_{c}(t)
\end{array}\right)\right]
$$

Analysis periodical signals $\mathrm{t}$ can be implemented by means of Fourier analysis over a sliding window of one cycle of the specified frequency that is used for processing of three input signals $x_{k}(t)$. Harmonics phasors derived from a windowed Fourier transform are functions of time due to sliding a window by different fragments of a signal with a complex form. At the beginning of transient process the current's spectrum consists of a DC - component, odd and even harmonics while in a steady state it has only odd components. Because spectrum of currents $i_{a}(t), i_{b}(t), i_{c}(t)$ are different, a set of 3-phase signals of each harmonic $k$ is unbalanced and can be decomposed into symmetrical components $i_{k}^{(+)}(t), i_{k}^{(-)}(t)$ and $i_{k}^{(0)}(t)$ characterized by time-dependent phasors $\dot{I}_{m k}^{(+)}(t), \quad \dot{I}_{m k}^{(-)}(t)$, $\dot{I}_{m k}^{(0)}(t)$ which are outputs of 3-phase sequence analyzer.

In the protection device (Fig.1) the analysis of input signals $\overline{i_{1}}, \overline{i_{2}^{\prime}}$ and differential signal $\overline{i_{d}}=\overline{i_{1}}-\overline{i_{2}^{\prime}}$ has been carried out on the basis of their decomposition into series of generalized symmetrical components of positive $i^{(+)}(t)$, negative $i^{(-)}(t)$ and zero $i^{(0)}(t)$ sequences
[15]. These symmetrical components were calculated for the first harmonic of current by means of discrimination of firs harmonic by low-pass filter and compensating elements implementing phase shift $\pm 120^{\circ}$. Transfer factor of phase-shifting link is calculated as:

$$
H_{\phi}\left(f, K_{\phi}\right)=-0.5+K_{\phi} \cdot \frac{1-j \frac{f}{f_{1}}}{1+j \frac{f}{f_{1}}}
$$

where $f_{1}=50 \mathrm{~Hz}$ - circuit voltage frequency.

Phase shift at frequency $f_{1}$ for $K_{\phi}=-0.5 \sqrt{3}$ is equal to $120^{\circ}$. For $K_{\phi}=0.5 \sqrt{3}$ phase shift is equal to $-120^{\circ}$.

Transfer factor of low-pass filter is calculated as:

$$
H(f)=\frac{-4}{\left(1+j \frac{f}{f_{1}}\right)^{4}}
$$

Fig. 4 shows instaneous positive component of differential current in case of short-circuit of $7 \%$ of turns in transformer secondary winding calculated by means of SimPowerSystem «3-phase sequence analyzer» and described filters. Diagram shows what both methods provide the same result for interval $t>0,02 \mathrm{~s}$ and described filters can be implemented in protection devices for calculation of symmetrical components of currents. Aperiodical component of currents can be filtered by means of rejection filter.

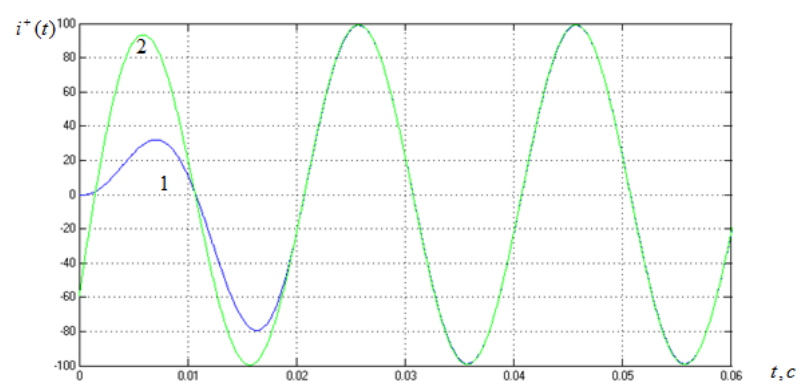

Fig. 4. Instaneous positive component of differential current in case of short-circuit of $7 \%$ of turns in transformer secondary winding calculated by means of SimPowerSystem «3-phase sequence analyzer» (1) and by low-pass filter (2).

\section{Results}

Comparative analysis of positive and negative sequences of a differential current has been carried out for the following modes: short circuiting of $W_{2 s c}$ turns of the secondary winding $W_{2}$ in the range $\beta=W_{2 s c} / W_{2}=0.01,0.02,0.05,0.1$, energizing a no loaded transformer, increment of input voltage, phase-to-phase short circuiting outside the protected zone. It was found that the ratio of amplitudes of 
negative $I_{m}^{(-)}$and positive $I_{m}^{(+)}$sequences is sensitive to the type of 3-phase asymmetry.

Results of simulation (Fig.5) demonstrate that an internal fault such as short-circuiting 5\% turns of the secondary winding leads to asymmetrical 3-phase differential currents $i_{d a}, i_{d b}, i_{d c}$ in contrast to the form of a healthy transformer excitation current which is a part of an unbalanced differential current.

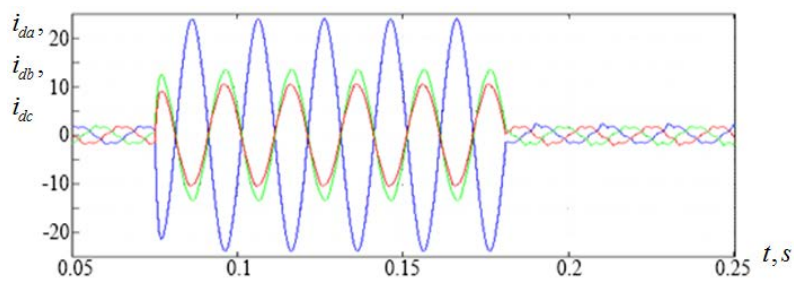

Fig. 5. Three-phase differential currents due to turn-to-turn short-circuiting $5 \%$ of the secondary winding in the background of healthy transformer excitation currents.

Fig.5 shows instantaneous curves of negative and positive components of a differential current (Fig. 6) and changing of amplitudes of corresponding sequences resulted from internal short circuiting (Fig.7). As it is seen from Fig. 6 the negative sequence component (curve 2) of the unbalanced differential current is negligibly small as compared to a positive one (curve 1). This is due to a filtering effect of decomposing the current into symmetrical components which results in changing the number of harmonics in compared currents from $k=1,3,5,7 \ldots$ to $k=5,11,17 \ldots$ and this leads to an appropriate decrease in RMS value.

Fig. 7 shows that amplitudes of negative and positive components are practically equal so that $\widehat{I}_{m}^{(-)}=I_{m}^{(-)} / I_{m}^{(+)} \approx 1$. This value of ratio $\widehat{I}_{m}^{(-)}$keeps valid if the number of short circuited turns is more than two percent, i.e. if $\beta \geq 0.02$.

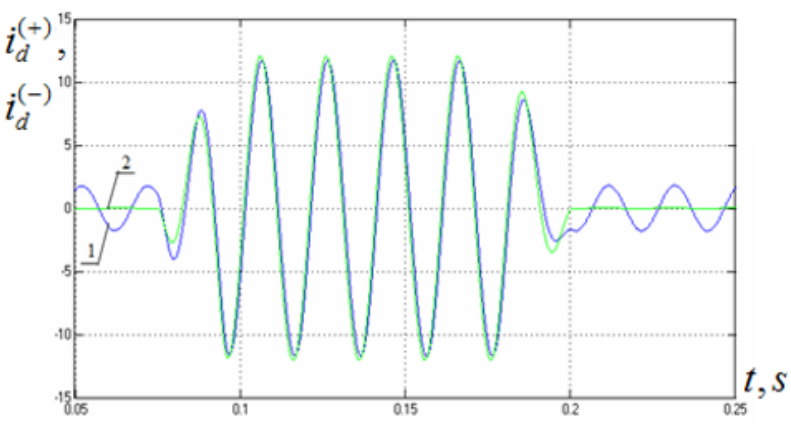

Fig. 6. Instantaneous current's forms of positive (1) and negative (2) sequences change because of internal short circuiting.

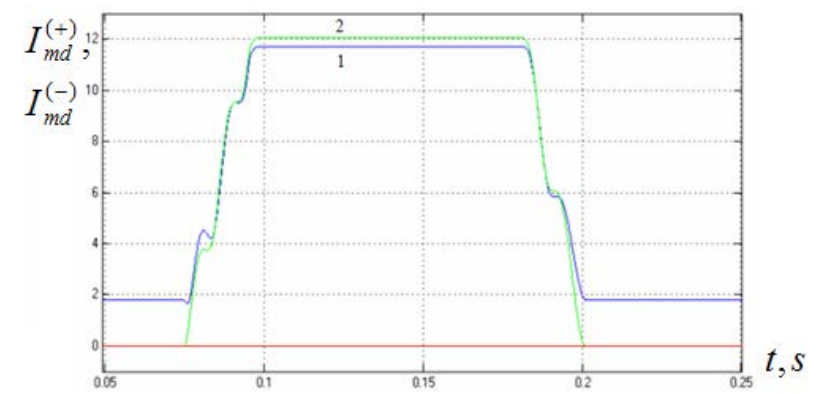

Fig. 7. Instantaneous current's amplitudes of positive (1) and negative (2) sequences change because of internal short circuiting.

Results of simulation for other modes of the transformer operation in the form used in Fig. 4 and Fig.5 have shown that relationship $\widehat{I}_{m}^{(-)} \approx 1$ is not the case for false differential currents. For instance, for inrush currents we have $\widehat{I}_{m}^{(-)} \leq 0.5$. The inequality $\widehat{I}_{m}^{(-)} \prec 1$ in varying degrees holds for other types of false differential currents: in case of external fault $\widehat{I}_{m}^{(-)} \leq 0.4$, in case of $30 \%$ increment of input voltage $\widehat{I}_{m}^{(-)} \leq 0.2$.

To use the obtained results for internal fault identification it is helpful to refer to a complex informative parameter expressed in the form:

$$
G\left(\widehat{I}_{m}^{(-)}\right)=\frac{1-\left(\widehat{I}_{m}^{(-)}\right)^{2}}{1+\left(\widehat{I}_{m}^{(-)}\right)^{2}}
$$

Dynamics of the parameter behavior during different kinds of faults is shown in Fig. 8.

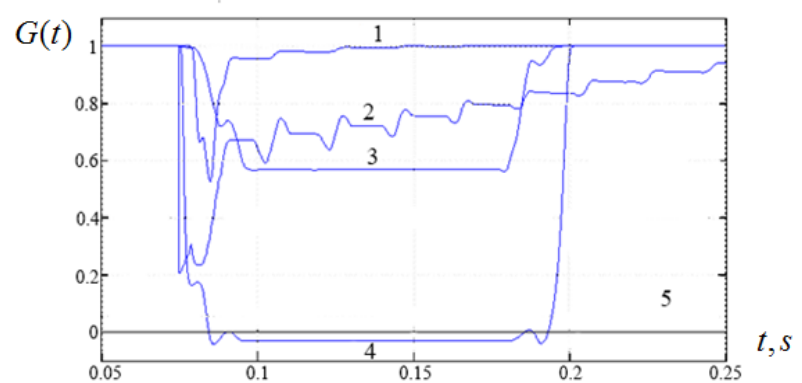

Fig. 8. A threshold value (5) and amplitude criteria behaviour for the transformer over-voltage (1), transformer energizing (2), external fault (3), inter-turn fault in the transformer winding (4).

Examination of curves allows identifying two regions of the relay operation relatively to a threshold value $S_{i}=0.05$. An area of the relay operation due to internal faults is defined by a condition $G\left(\bar{I}_{m}^{(-)}\right)<S_{i}$. Another area $G\left(\widehat{I}_{m}^{(-)}\right)>S_{i}$ corresponds to false differential currents caused by external faults, inrush currents, transformer over excitation etc. In this case relay operation is to be prevented. 


\section{Discussion}

The paper deals with analysis of the power transformer's fault currents under different conditions of operation using Simulink MATLAB. Analysis was carried out on the basis of decomposition of analyzed currents to generalized symmetrical components by means of a sequence analyzer which output signals in the form of dynamic phasors of each harmonic which were used to reconstruct instantaneous values of currents' positive, negative and zero sequences.

Low-pass filter with correction link was proposed for calculation of symmetrical components of first harmonic of differential current. Comparative analysis of dynamic phasors shows the sensitivity of amplitudes of negative and positive sequences of differential currents to fault location and allows formulation of algorithm for detecting internal faults in the transformer windings.

\section{References}

1.A. Guzmán, N. Fischer, C. Labuschagne, J. Rel. Pow. 2(3), 19-35 (2011)

2.B. N. Abramovich, Y. A. Sychev, R. Y. Zimin Proc. 2019 IEEE Conf. Russ. Young Res. Electr. Electron. Eng. ElConRus 401-406 (2019)

3. Y. V. Gulkov, D. A. Ustinov, Int. J. Appl. Eng. Res. 12(9), 1127-1130 (2017).

4.O. M. Bolshunova, A. A. Korzhev, and A. M. Kamyshyan, IOP Conf. Ser. Mater. Sci. Eng. 327, 052007 (2018)

5. A. N. Skamyin, O. S. Vasilkov, PQ and SEEM 2019, 18957601 (2019)

6.H. Abniki, A. Majzoobi, H. Monsef, H. Dashti, H. Ahmadi, P. Khajavi, Proc. Int. Sym.: Mod. Electr. Pow. Sys. (MEPS), 1-6 (2010)

7.Z. Gajić, I. Brnčić, B. Hilström, F. Mekić, I. Ivanković, Proc. 32nd Ann. West. Prot. Rel. Conf., 1-20 (2005).

8.A. Guzmán, S. Zocholl, H. Altuve, SEL Paper, 162-171 (2000)

9. S.B. Parmar, B.S.Shah. Int. J. Nov. Res. Dev. 2(5), 108-111 (2017)

10. K. Ramesh, M. Sushama. Intl. Conf. Adv. El. Eng, 1-7 (2014)

11. N. Farzin, M. Vakilian, E. Hajipour. IEEE Conf. Pow. En. Soc. 34(2), 700-709 (2019)

12. N.C. Joshi, R.K. Jarial, Y.R. Sood, R. Thapliyal, MIT Int. J. El. Instr. Eng., 2(2), 77-81 (2012)

13. R.S. Bhide, M.S.S. Sreenivas, A. Banerjee, R. Somakumar, Proc. ICSET 2010, (2010)

14. G.C. Paap, IEEE Trans. Pow. Sys. 15(2), 522-528 (2000)

15. M.T. Haque, S.H., Sec. Int. Conf. Electric. Electron. Eng. ELECO’2001, 152-156 (2001) 